\title{
Securing linked transportation systems: economic implications and investment strategies
}

\author{
Adib Kanafani • Jiangchuan Huang
}

Received: 19 July 2010 /Accepted: 13 September 2010 /Published online: 23 September 2010

(C) The Author(s) 2010. This article is published with open access at Springerlink.com

\begin{abstract}
The security of the transportation system depends on that of any of its components and how they are interlinked. But the securing of each component is oftentimes in the hand of the agency in whose jurisdiction it falls. Literature on reliability and security economics suggests that when security is defined by the weakest link in an interlinked system, then its level is determined by the agent with the highest cost-benefit ratio, and the other agents have the tendency to under-invest or free ride. When security is a function of total effort, then the opposite obtains and the reliability will depend on the agent with the lowest cost-benefit ratio. These conditions arise in urban transportation. This research explores agency investment behavior in multi-agency urban transportation systems develops guidelines for investments in security. The question to answer: is it preferable to let each agency operate its own security budget and make its own investment decisions or is this process better centralized?
\end{abstract}

Keywords Transportation network security Shared node and exclusive node dominated network $\cdot$ Mixed network $\cdot$ System reliability

\section{Introduction}

Understanding the vulnerability of transportation networks has taken on a heightened urgency following the events of the last decade, as Government agencies scramble to develop investment strategies to protect these networks from security threats. The little we know about transportation security suggests increased

Research supported by a grant from the University of California Transportation Center.

\footnotetext{
A. Kanafani $(\bowtie) \cdot$ J. Huang

University of California, Berkeley, CA, USA

e-mail: kanafani@berkeley.edu

J. Huang

e-mail: jiangchuan@berkeley.edu
} 
vulnerability at points of inter-modal connection or at large scale termini, where two conditions arise: large numbers of people making transfers and an institutional ambiguity regarding who is responsible for securing the system. This ambiguity can result in unbalanced and inefficient security investment. For example while large amounts of money are spent on securing an airport, little or nothing, is done to secure the shuttle buses that penetrate deep into the airport infrastructure!

Current literature on the vulnerability of transportation systems focuses mainly on the problem of physical topological deficiency and deals with the loss of particular links or nodes in a network. Du and Nicholson (1997) measure vulnerability using performance of the transportation network. Berdica (2002) analyzes vulnerability from a perspective of reliability of connectivity, travel time and capacity, which he calls serviceability. Jenelius et al. (2006) uses graph theory to depict exposure, criticality and importance. Nagurney and Qiang (2007) define an efficiency measure to assess the performance of a transportation network.

Little has been done on how to reduce vulnerability or to allocate security investment between transportation agencies and among the different components of the transportation network. But the literature on reliability economics does provide a way of thinking about the problem of transportation network security. The framework of investment in public goods has been used for this considering that security is in many respects a public good. Hirshleifer (1983) considered the marginal cost and marginal return of each individual involved in the provision of public good and defined conditions for a Nash equilibrium. Varian (2004), in his work on information system reliability built on Hirshleifer's work, defined three prototypical cases in the context of system reliability: (a) Total effort, where reliability depends on the sum of the efforts exerted by the individuals, (b) Weakest link, where reliability depends on the minimum effort, and (c) Best shot, where reliability depends on the maximum effort. He analyzed the Nash equilibrium and social optimum of the utility of individuals with different costbenefit for each case.

In this paper we analyze the endogenous and exogenous features of transportation network that affect the security investment behavior of agencies and thus the security of the transportation network. We also analyze the effect of the knowledge of the attacker to different network types. We aim to extend Varian's work to the level of a transportation network, of which different components come under the jurisdiction of different agencies. We map two of the three prototypical cases mentioned above to the particular transportation networks, with the total effort case applying to a shared node dominated network and the weakest link case to a perfect information exclusive node dominated network. We also create a combination of total effort and weakest link applying to the imperfect information exclusive node dominated network and the mixed network. We draw a comparison between the Nash equilibrium and social optimal, as defined by the utility of the actors and security level of the system in each case and show that social optimal is always preferable to Nash equilibrium. Finally, we give two examples of two-agency mixed network, one with similar investment incentive and the other with different investment incentives with application to the security of airport-shuttle bus operator system. We conclude with some policy recommendations regarding the organization of investment in system security. 


\section{Definitions and concepts}

Transportation facilities that make up a complex transportation network often come under the jurisdiction of different transportation agencies. Questions regarding the following arise when more than one agency is involved in investment on security: (a) the incentive of each agency to invest; (b) the effectiveness of each agency to invest; (c) the relationship between the actions of different agencies; and (d) the attacker's knowledge of the level of security in each node. The first and second questions can be dealt with in a cost-benefit analysis (an endogenous agency feature), the third and fourth questions require an analysis of the type of transportation network the agencies are involved in (an exogenous system feature) based on a perfect or imperfect information assumption separately.

Agency feature (endogenous)

We define a taxonomy of agencies involved in the transportation system based on two criteria: whether they are motivated to invest in the security of the system, and whether they are effective in doing so. The resulting four categories are shown in the Table 1.

We assume the following behavior for each category as shown below, with some examples for each:

1. Motivated and Effective $(++)$ : this agency will invest in securing its system themselves. Examples include Pentagon, White House

2. Motivated but Not Effective $(+-)$ : pay other effective agencies to invest, e.g. airport on the security of shuttle bus

3. Not Motivated but Effective $(-+)$ : paid by other motivated agencies to invest, e.g. shuttle bus operator for an airport

4. Not Motivated and Not Effective (- $)$ : not involved check the wording here!

In which category an agency falls in is mostly determined by its intrinsic feature, partially determined by the part to secure and the current security level of the facility. For example, the airport is effective to secure its facilities while it has no way to secure the shuttle buses owned by another agency that penetrate deep into the airport infrastructure. Also, when the security level of an agency is already very high, it is not motivated or effective to invest any more. Thus each agency invests on the security of the system with different investment incentive and investment effectiveness.

Table 1 Different agency behavior according to different motivation and effectiveness.

\begin{tabular}{|c|c|c|c|}
\hline & & \multicolumn{2}{|l|}{ Effective } \\
\hline & & Yes & No \\
\hline \multirow[t]{2}{*}{ Motivated } & $\mathrm{Y}$ & Invest themselves & Pay other effective agencies to invest \\
\hline & $\mathrm{N}$ & Paid by other motivated agencies to invest & Not involved \\
\hline
\end{tabular}




\section{System feature (exogenous)}

The transportation system is composed of nodes (stations, airports, ports) and links (roads, subway lines, air routes). Each node or link may be under the jurisdiction of one (exclusively owned) or more (shared) agencies. The securing of shared nodes is subject to investment decisions made by the agencies that have jurisdiction over them. For example, railway and highway agencies may be involved in the securing of a same bridge they share. Exclusive system components may come under the jurisdiction of one agency, even though their operation may involve intermodal connections that involve systems that come under the jurisdiction of other agencies. For example, a shuttle bus operator may have the sole responsibility to secure its buses that connect between a city terminal and an airport.

In this context we can think of shared node dominated networks, exclusive node dominated networks and mixed networks. In a shared node dominated network, most of the nodes are shared and the lack of investment by one agency will not necessarily result in increased vulnerability since other agencies may decide to invest in the system. In an exclusive node dominated network, each node is exclusively owned by one agency and the absence of investment by the cognizant agency will lead to increased vulnerability. In a mixed network, there are significant amounts of both shared and exclusive nodes and the network will have the common features of the former two network types.

\section{The attacker's knowledge (counterpart)}

In a shared node dominated network, the attacker's knowledge of the current security level of each nodes does not quite matter in the system security and the invest incentive of agencies. Since almost all nodes are shared nodes, if some agencies fail to invest, other agencies can still invest in the shared nodes and sustain a high security level of the system. The attackers will always find themselves in a situation where almost all the nodes are in the same security level. Knowledge of the security level of each agency does not help them make decision, they just pick up one node randomly to attack. On the other side, the agencies, knowing that the knowledge of the attacker on their decision of investments on the security is of little use to the attacker, make decision without considering whether the attacker knows their decision or not.

In an exclusive node dominated network, however, the attacker's knowledge of the current security level of each nodes can make big difference in the system security and the invest incentive of agencies. Since exclusive node dominated network can be deficient in certain part if some agencies fail to invest, if the attacker knows the security level of each nodes, they will always attack the nodes belong to the agency with the fewest investment, rendering the additional investment of other agencies on their nodes useless. If the attacker does not know the security level of each node, they will attack randomly, with a probability of attack on each node. This lead to the discussion of perfect information and imperfect information of the attacker on exclusive node dominated network.

Our interest in this paper is in shared node networks and exclusive node networks in different information set of the attacker and their common features adaptable to mixed networks, and in the interplay between investment decisions of multiple agencies. 
Utility function

Following Varian (2004) we adopt the following utility function to describe an agency's goal of securing its system at minimum cost:

$$
U_{i}=P\left(x_{1}, x_{2}, \cdots, x_{n}\right) v_{i}-c_{i} x_{i}, \quad i=1,2, \cdots, n
$$

where:

$\begin{array}{ll}U_{i} & \text { is the utility of agency } i . \\ x_{i} & \text { is the level of effort expended by agency } i \text { in system security. } \\ P\left(x_{1}, x_{2}, \cdots, x_{n}\right), & \text { is the security function-a measure of the resulting level of } \\ & \text { security, such as the probability that the system is secured. } P(\cdot) \text { can } \\ & \text { take different forms depending on the relationship between } \\ & \text { agencies. } \\ & \text { is the utility received by agency } i \text { if the system is secured. It } \\ & \text { measures an agency's motivation or incentive to enhance the } \\ & \text { security. } \\ & \text { is the cost to exert one unit of effort. It measures an agency's } \\ & \text { effectiveness to enhance the security- the smaller } c_{i}, \text { the more } \\ & \text { effective agency } i \text { in securing its system. We can use } c_{i} / v_{i} \text {, the cost- } \\ & \text { benefit ratio of agency } i \text { as a comparative indicator of incentive and } \\ & \text { effectiveness for security investment. }\end{array}$

The following properties of the utility function: $U_{i}=P\left(x_{1}, x_{2}, \cdots, x_{n}\right) v_{i}-c_{i} x_{i}$ are fairly obvious:

1. Nonnegative: $x_{i} \geq 0, v_{i} \geq 0, c_{i} \geq 0, P\left(x_{1}, x_{2}, \cdots, x_{n}\right) \geq 0$

2. Diminishing investment incentive: $P\left(x_{1}, x_{2}, \cdots, x_{n}\right)$ is $C^{1},\left.\frac{\partial P}{\partial x_{i}}\right|_{x_{i}^{-}}$is nonnegative and decreasing on $x_{i}$, where $x_{i}^{-} \triangleq\left(x_{1}, x_{2}, \cdots, x_{i-1}, x_{i+1}, \cdots, x_{n}\right)$

3. Relative investment advantage: $\frac{\partial P}{\partial x_{i}}>\frac{\partial P}{\partial x_{j}}$ when $x_{i} \ll x_{j}$

4. Investment motivations: Agency $i$ is motivated to invest on security when $\frac{\partial U_{i}}{\partial x_{i}}=v_{i} \frac{\partial P}{\partial x_{i}}-c_{i}>0$, i.e. marginal return $M R=v_{1} \frac{\partial P}{\partial x_{1}}>$ marginal cost $M C=c_{1}$. Let $G(\cdot)$ be the inverse of $P^{\prime}(\cdot)$, define Individual investment optimum $x_{i}^{*}=G\left(\frac{c_{i}}{v_{i}}\right)$, thus $x_{i}^{*}$ satisfies $v_{i} \frac{\partial P}{\partial x_{i}}=c_{i} \Leftrightarrow \frac{\partial U_{i}}{\partial x_{i}}=v_{i} \frac{\partial P}{\partial x_{i}}-c_{i}=0$.

Then $\frac{\partial U_{i}}{\partial x_{i}}>0$ when $x_{i}<x_{i}^{*}, \frac{\partial U_{i}}{\partial x_{i}}<0$ when $x_{i}>x_{i}^{*}$. This implies Claim 1:

Claim 1: Under free will, one agency wants to match its investment to its individual optimum, it will never invest in its negative incentive zone. That is, the agency will never invest more than $x_{i}^{*}$.

\section{Mapping}

In this section we develop the security investment models for a number of situations that might arise in real world transportation systems. We first consider the case of a 
shared node dominated network in which the security of the system depends on the total effort expended by all agencies involved. We then consider the case of an exclusive node dominated network in which each agency decides separately on its security investment and in which the attacker has perfect information about the level of security in each node, thus making this a case of weakest-link vulnerability. We follow that with a case representing a combination of these conditions. We conclude the mapping in Table 2.

Shared node dominated network-Total effort

In a shared node dominated network, any agency can invest in any of the nodes, the efforts are substitutable and cumulative, i.e. $\frac{\partial P}{\partial x_{i}}=\frac{\partial P}{\partial x_{j}}$ for all $x_{i} \geq 0, x_{j} \geq 0$, making this a total effort case as defined in Varian (2004) with the security function:

$$
P\left(x_{1}, x_{2}, \cdots, x_{n}\right) \triangleq P\left(\sum_{i=1}^{n} x_{i}\right)
$$

Without loss of generality, we look at the case of two agencies, $1 \& 2$, both of which can invest in any node of the network. The diminishing margin of $P\left(x_{1}, x_{2}, \cdots, x_{n}\right)$ implies that if agency 2 invests more, agency 1's investment is squeezed in the diminished part, causing a reduction of incentive and resulting in free riding. An illustration is shown in Figure 1a, where 1,2 means both agency 1 and 2 can invest in the node. The isoquant map of security function $P$ is shown in Figure 1b, which illustrates the diminishing returns of $P$.

Let's say agency 1 has a higher cost-benefit ratio, $c_{1} / v_{1}>c_{2} / v_{2}$, then agency 2 has higher investment incentive than agency 1 (either has higher security incentive, higher $v_{2}$; or is more effective, lower $c_{2}$ ). For the sake of reader, we recap Varian's model for Nash equilibrium and social optimum with extensions of our own understanding. Note that $x_{i}^{N}, U_{i}^{N}, U^{N}, P^{N}$ are the effort and utility of agency $i$, the utility of the system and the security level of Nash equilibrium (when agencies choose to plan individually). Similarly, $x_{i}^{S}, U_{i}^{S}, U^{S}, P^{S}$ are the same quantities of social optimum (when agencies choose to plan coordinately).

Table 2 Map from network types to reliability prototypical cases in different information set.

\begin{tabular}{lll}
\hline & \multicolumn{2}{c}{ The attacker's knowledge of the level of security in each node } \\
\cline { 2 - 3 } & Perfect information & Imperfect information \\
\hline $\begin{array}{l}\text { Shared node dominated network } \\
\text { Exclusive node dominated network }\end{array}$ & $\begin{array}{l}\text { Total effort } \\
\text { Weakest link }\end{array}$ & $\begin{array}{l}\text { Total effort } \\
\text { Combination of total effort and weakest } \\
\text { link }\end{array}$ \\
\hline
\end{tabular}


a

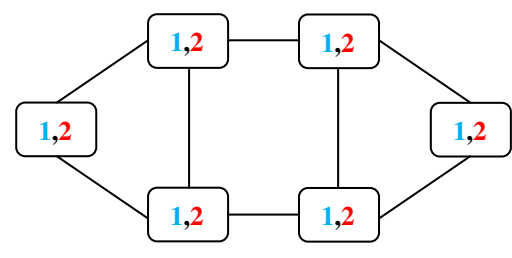

b

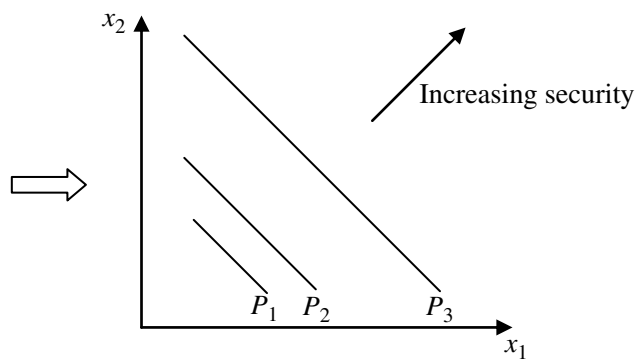

Figure 1 a Shared node dominated network b Isoquant map of security function

\section{Nash equilibrium}

Agency 1 makes decision (chooses $x_{1}$ ) with respect to agency 2's decision $\left(x_{2}\right)$ by solving: $\left.\max _{x_{1}} U_{1}\right|_{x_{2}}$

$$
\text { i.e. } \max _{x_{1}} v_{1} P\left(x_{1}+x_{2}\right)-c_{1} x_{1}
$$

Let $G(\cdot)$ be the inverse of $P^{\prime}(\cdot)$, then it is decreasing. From claim 1, we know the individual investment optimum of agency $1 \& 2$ are $x_{1}^{*}=G\left(\frac{c_{1}}{v_{1}}\right)$ and $x_{2}^{*}=G\left(\frac{c_{2}}{v_{2}}\right)$, neither of them choose to invest more than $x_{1}^{*}$ and $x_{2}^{*}$.

Take first order condition: $v_{1} P^{\prime}\left(x_{1}+x_{2}\right)-c_{1}=0$, we get agency 1 's decision $\left(x_{1}\right)$ with respect to agency 2 's decision $\left(x_{2}\right)$ :

$$
x_{1}=\max \left\{G\left(\frac{c_{1}}{v_{1}}\right)-x_{2}, \quad 0\right\}=\max \left\{x_{1}^{*}-x_{2}, \quad 0\right\}
$$

Similarly, agency 2's decision $\left(x_{2}\right)$ with respect to agency 1's decision $\left(x_{1}\right)$ is:

$$
x_{2}=\max \left\{G\left(\frac{c_{2}}{v_{2}}\right)-x_{1}, \quad 0\right\}=\max \left\{x_{2}^{*}-x_{1}, \quad 0\right\}
$$

The two decision curves only have one intersection point, this is the Nash equilibrium, where $x_{1}^{N}=0, x_{2}^{N}=G\left(\frac{c_{2}}{v_{2}}\right)$. Since the efforts are substitutable and cumulative, agency 2 , with higher investment incentive, fulfills its obligation by investing at its individual optimum $x_{2}^{*}=G\left(\frac{c_{2}}{v_{2}}\right)$ while agency 1 frees rides. In a word, the security is determined by the most motivated or effective agency (with the lowest cost-benefit ratio), others free ride. This is shown in Figure 2.

\section{Social optimum}

We want to maximize the utility of the system by solving $\max _{x_{1}, x_{2}}\left(U_{1}+U_{2}\right)$

$$
\text { i.e. } \max _{x_{1}, x_{2}}\left(v_{1}+v_{2}\right) P\left(x_{1}+x_{2}\right)-c_{1} x_{1}-c_{2} x_{2}
$$

if $c_{1}<c_{2}$, this reduces to $\max _{x_{1}, x_{2}=0}\left(v_{1}+v_{2}\right) P\left(x_{1}\right)-c_{1} x_{1}$

Take first order condition: $\left(v_{1}+v_{2}\right) P^{\prime}\left(x_{1}\right)-c_{1}=0$ 
Figure 2 Nash equilibrium and social optimum of total effort

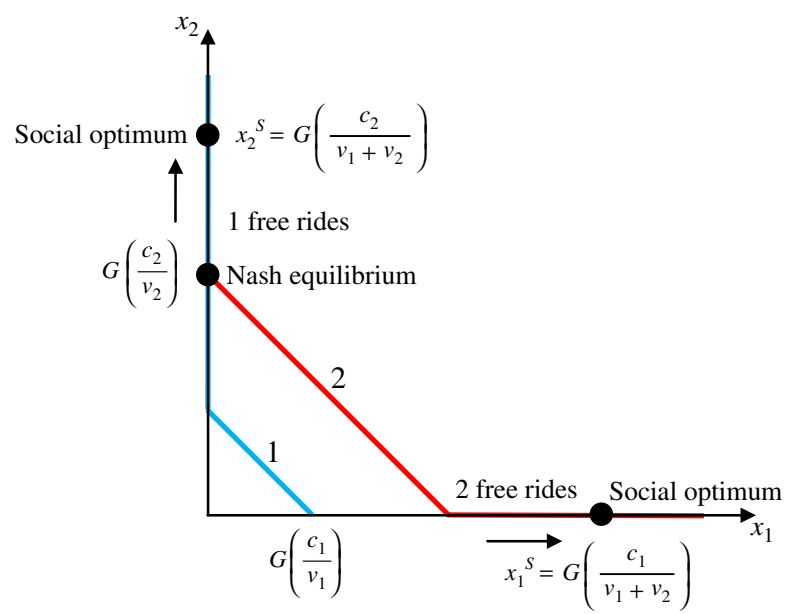

We get social optimum when $x_{1}^{S}=G\left(\frac{c_{1}}{v_{1}+v_{2}}\right), x_{2}^{S}=0$ for $c_{1}<c_{2}$

Similarly, We get social optimum when $x_{1}^{S}=0, x_{2}^{S}=G\left(\frac{c_{2}}{v_{1}+v_{2}}\right)$ for $c_{1}>c_{2}$

We have $x_{1}^{S}=G\left(\frac{c_{1}}{v_{1}+v_{2}}\right) G\left(\frac{c_{1}}{v_{1}}\right)=x_{1}^{*}$ and $x_{2}^{S}=G\left(\frac{c_{2}}{v_{1}+v_{2}}\right) G\left(\frac{c_{2}}{v_{2}}\right)=x_{2}^{*}$.

While the agency with the lowest cost exerts enough efforts for itself, there is still marginal benefit for the system. The system demand the lowest-cost agency to invest in its negative incentive zone, that is, to invest more than its individual optimum, which is a violation of claim 1 . The lowest-cost agency is not willing to cooperate for social optimum. Other agencies could give the lowest-cost agency money to offset the difference between individual and social optimum. Alternatively, the government could give the lowest-cost agency some subsidy to entice it to invest at social optimum.

To sum up, we have the investment strategies of agency $1 \& 2$ and the outcomes as shown in Table $3\left(c_{1}<c_{2}\right)$ and Table $4\left(c_{1}>c_{2}\right)$.

At the Nash equilibrium, the level of security is determined by the agency with the lowest cost-benefit ratio and the other agencies free ride. But at the social optimum the security level is determined by the agency with the lowest cost, and the

Table 3 Nash equilibrium and social optimum of shared node dominated network $c_{1} / v_{1}>c_{2} / v_{2}, c_{1}<c_{2}$.

\begin{tabular}{lll}
\hline Nash equilibrium & & Social optimum \\
\hline$x_{1}^{N}=0, x_{2}^{N}=G\left(\frac{c_{2}}{v_{2}}\right)$ & $x_{1}^{S}=G\left(\frac{c_{1}}{v_{1}+v_{2}}\right), x_{2}^{S}=0$ \\
$P^{N}=P\left(G\left(\frac{c_{2}}{v_{2}}\right)\right)$ & $<$ & $P^{S}=P\left(G\left(\frac{c_{1}}{v_{1}+v_{2}}\right)\right)$ \\
$U_{1}^{N}=v_{1} P\left(G\left(\frac{c_{2}}{v_{2}}\right)\right)$ & $>$ & $U_{1}^{S}=v_{1} P\left(G\left(\frac{c_{1}}{v_{1}+v_{2}}\right)\right)-c_{1} G\left(\frac{c_{1}}{v_{1}+v_{2}}\right)$ \\
$U_{2}^{N}=v_{2} P\left(G\left(\frac{c_{2}}{v_{2}}\right)\right)-c_{2} G\left(\frac{c_{2}}{v_{2}}\right)$ & $<$ & $U_{2}^{S}=v_{2} P\left(G\left(\frac{c_{1}}{v_{1}+v_{2}}\right)\right)$ \\
$U^{N}=\left(v_{1}+v_{2}\right) P\left(G\left(\frac{c_{2}}{v_{2}}\right)\right)-c_{2} G\left(\frac{c_{2}}{v_{2}}\right)$ & $<$ & $U^{S}=\left(v_{1}+v_{2}\right) P\left(G\left(\frac{c_{1}}{v_{1}+v_{2}}\right)\right)-c_{1} G\left(\frac{c_{1}}{v_{1}+v_{2}}\right)$ \\
\hline
\end{tabular}


Table 4 Nash equilibrium and social optimum of shared node dominated network $c_{1} / v_{1}>c_{2} / v_{2}, c_{1}>c_{2}$.

\begin{tabular}{lll} 
Nash equilibrium & & Social optimum \\
\hline$x_{1}^{N}=0, x_{2}^{N}=G\left(\frac{c_{2}}{v_{2}}\right)$ & & $x_{1}^{S}=0, x_{2}^{S}=G\left(\frac{c_{2}}{v_{1}+v_{2}}\right)$ \\
$P^{N}=P\left(G\left(\frac{c_{2}}{v_{2}}\right)\right)$ & $<$ & $P^{S}=P\left(G\left(\frac{c_{2}}{v_{1}+v_{2}}\right)\right)$ \\
$U_{1}^{N}=v_{1} P\left(G\left(\frac{c_{2}}{v_{2}}\right)\right)$ & $<$ & $U_{1}^{S}=v_{1} P\left(G\left(\frac{c_{2}}{v_{1}+v_{2}}\right)\right)$ \\
$U_{2}^{N}=v_{2} P\left(G\left(\frac{c_{2}}{v_{2}}\right)\right)-c_{2} G\left(\frac{c_{2}}{v_{2}}\right)$ & $>$ & $U_{2}^{S}=v_{2} P\left(G\left(\frac{c_{2}}{v_{1}+v_{2}}\right)\right)-c_{2} G\left(\frac{c_{2}}{v_{1}+v_{2}}\right)$ \\
$U^{N}=\left(v_{1}+v_{2}\right) P\left(G\left(\frac{c_{2}}{v_{2}}\right)\right)-c_{2} G\left(\frac{c_{2}}{v_{2}}\right)$ & $<$ & $U^{S}=\left(v_{1}+v_{2}\right) P\left(G\left(\frac{c_{2}}{v_{1}+v_{2}}\right)\right)-c_{2} G\left(\frac{c_{2}}{v_{1}+v_{2}}\right)$
\end{tabular}

other agencies free ride. The social optimum results in a higher level of security $\left(P^{S}>P^{N}\right)$ and of system utility $\left(U^{S}>U^{N}\right)$.

Perfect information exclusive node dominated network-Weakest link

In an exclusive node dominated network, only one agency can invest in a node, the investments are neither cumulative nor substitutable. Each agency will always find the need of investment for itself and the burden cannot be pushed to others. The failure of one agency will lead to large deficiency of the transportation system. All the agencies have the incentive to invest on their own nodes. As described below system vulnerability in this case depends on the weakest link, rather than on the total effort by all agencies.

Since exclusive node dominated network can be deficient in certain part if some agencies fail to invest, the attacker's knowledge of the current security level of each nodes can make a big difference in the system security and the invest incentive of agencies. This lead to the discussion of perfect information and imperfect information of the attacker on exclusive node dominated network.

If the attacker knows the current security level of the nodes in an exclusive node dominated network, then they will always attack through the most vulnerable node, the security of the network is determined by the agency that exerts fewest effort, i.e. $\frac{\partial P}{\partial x_{i}}>\frac{\partial P}{\partial x_{j}}=0$ when $x_{i} \ll x_{j}$. The security function takes the form:

$$
P\left(x_{1}, x_{2}, \cdots, x_{n}\right) \triangleq \min \left\{P\left(x_{1}\right), P\left(x_{2}\right), \cdots, P\left(x_{n}\right)\right\}=P\left(\min \left\{x_{1}, x_{2}, \cdots, x_{n}\right\}\right)
$$

The equality holds because $P\left(x_{i}\right)$ is monotonically increasing.

The network type and the isoquant map of security function $P$ are shown in Figure $3 \mathrm{a}$ and $\mathrm{b}$ for the case of two agencies, where 1 means only agency 1 can invest in the node and 2 means only agency 2 can invest in the node.

Using the same assumption $\left(c_{1} / v_{1}>c_{2} / v_{2}\right)$ and notation in Shared node dominated network-Total effort, We repeat Varian's model for Nash equilibrium and social optimum with extensions of our own understanding. 
a

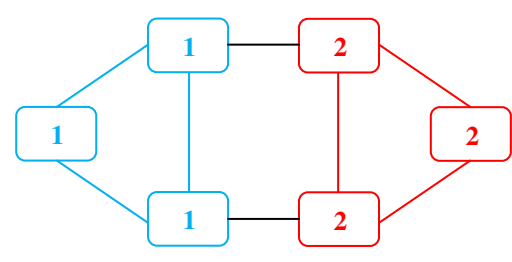

b

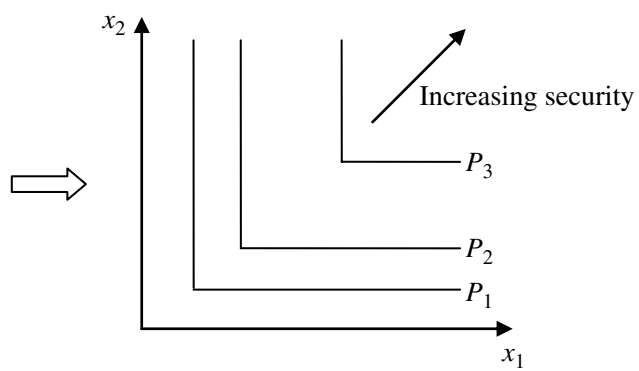

Figure 3 a Perfect Information exclusive node dominated network b Isoquant map of security function

\section{Nash equilibrium}

Agency 1 makes decision (chooses $\left.x_{1}\right)$ with respect to agency 2's decision $\left(x_{2}\right)$ by solving: $\left.\max _{x_{1}} U_{1}\right|_{x_{2}}$

$$
\text { i.e. } \max _{x_{1}} v_{1} P\left(\min \left\{x_{1}, x_{2}\right\}\right)-c_{1} x_{1}
$$

(i) If $x_{1}<x_{2}, \max _{x_{1}} v_{1} P\left(x_{1}\right)-c_{1} x_{1}$

Take first order condition: $v_{1} P^{\prime}\left(x_{1}\right)-c_{1}=0 \Rightarrow x_{1}=G\left(\frac{c_{1}}{v_{1}}\right) x_{2}$

(ii) If $x_{1} \geq x_{2}, \max _{x_{1}} v_{1} P\left(x_{2}\right)-c_{1} x_{1}$, agency 1 sets $x_{1}$ as small as possible, thus $x_{1}=x_{2}$

We get agency 1's decision $\left(x_{1}\right)$ with respect to agency 2's decision $\left(x_{2}\right)$ :

$$
x_{1}=\min \left\{G\left(\frac{c_{1}}{v_{1}}\right), \quad x_{2}\right\}=\min \left\{x_{1}^{*}, x_{2}\right\}
$$

Similarly, agency 2's decision $\left(x_{2}\right)$ with respect to agency 1's decision $\left(x_{1}\right)$ is:

$$
x_{2}=\min \left\{G\left(\frac{c_{2}}{v_{2}}\right), \quad x_{1}\right\}=\min \left\{x_{2}^{*}, x_{1}\right\}
$$

As Varian observed, there are a whole range of Nash equilibria. The largest of these is at $x_{1}^{N}=x_{2}^{N}=\min \left\{G\left(\frac{c_{1}}{v_{1}}\right), G\left(\frac{c_{2}}{v_{2}}\right)\right\}$. This Nash equilibrium Pareto dominates the others, so it is natural to think of it as the likely outcome (Varian 2004).

Since agency 1 has a higher cost-benefit ratio by assumption and $G$ is decreasing, we have $G\left(\frac{c_{1}}{v_{1}}\right) G\left(\frac{c_{2}}{v_{2}}\right), x_{1}^{N}=x_{2}^{N}=G\left(\frac{c_{1}}{v_{1}}\right)$.

We could see that claim 1 holds in that agency $1 \& 2$ never choose to invest more than their individual investment optimum. Also, they never choose to invest more than the other one does. This is because the nodes exclusively owned by the agency with the fewest investment become the most vulnerable part of the network. The attacker, knowing this, will always attack the nodes belong to the agency with the fewest investment, rendering the additional investment of other agencies on their 
nodes useless. Thus other agencies choose to match their investment to that of the one with the fewest investment and of course not to surpass their own individual investment optimum. As a result, each agency matches their investment to the least individual investment optimum, $\min \left\{x_{1}^{*}, x_{2}^{*}, \cdots, x_{n}^{*}\right\}=G\left(\max \left\{c_{i} / v_{i}\right\}\right)$. In a word, the security is determined by the least motivated or effective agency (with the highest cost-benefit ratio), no one free ride. This is shown in Figure 4.

\section{Social optimum}

We want to maximize the utility of the system by solving $\max _{x_{1}, x_{2}}\left(U_{1}+U_{2}\right)$

$$
\text { i.e. } \max _{x_{1}, x_{2}}\left(v_{1}+v_{2}\right) P\left(\min \left\{x_{1}, x_{2}\right\}\right)-c_{1} x_{1}-c_{2} x_{2}
$$

It is obvious that $x_{1}=x_{2}$ at optimum, thus the problem reduces to

$$
\max _{x}\left(v_{1}+v_{2}\right) P(x)-\left(c_{1}+c_{2}\right) x
$$

Take first order condition: $\left(v_{1}+v_{2}\right) P^{\prime}(x)-\left(c_{1}+c_{2}\right)=0$ have

We get social optimum when $x_{1}^{S}=x_{2}^{S}=G\left(\frac{c_{1}+c_{2}}{v_{1}+v_{2}}\right)$. Since $G$ is decreasing, we

$$
x_{1}^{S}=x_{2}^{S}=G\left(\frac{c_{1}+c_{2}}{v_{1}+v_{2}}\right)>\min \left\{G\left(\frac{c_{1}}{v_{1}}\right), \quad G\left(\frac{c_{2}}{v_{2}}\right)\right\}=x_{1}^{N}=x_{2}^{N} .
$$

Follow our assumption, $x_{1}^{S}=x_{2}^{S}=G\left(\frac{c_{1}+c_{2}}{v_{1}+v_{2}}\right)>G\left(\frac{c_{1}}{v_{1}}\right)=x_{1}^{N}=x_{2}^{N}$

While at Nash equilibrium each agency matches their investment to the least individual investment optimum, there is still marginal benefit for the system. The system demand each agency to enhance their investment to the system investment optimum $G\left(\sum_{i=1}^{n} c_{i} / \sum_{i=1}^{n} v_{i}\right)$. Agencies with lower individual investment optimum

Figure 4 Nash equilibrium and social optimum of weakest link

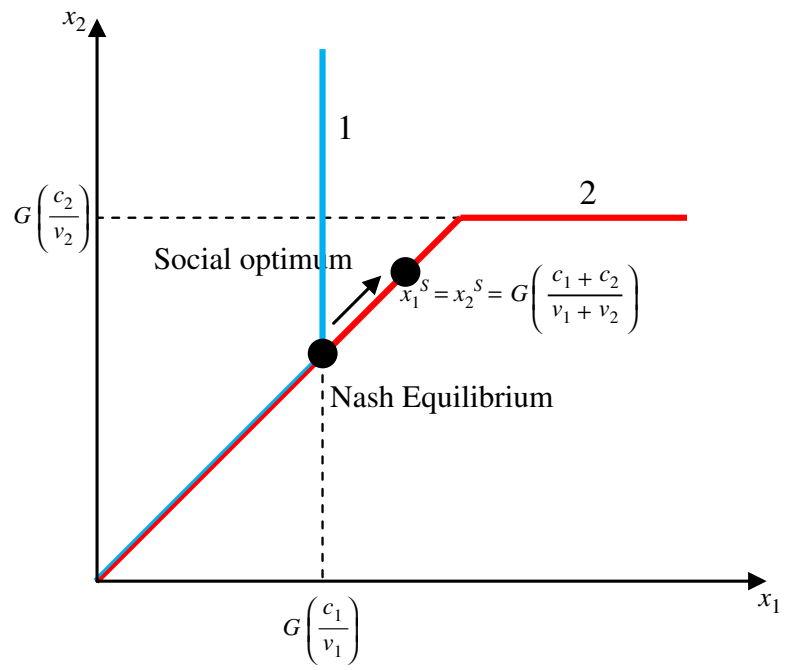


(with a higher cost-benefit ratio than $\sum_{i=1}^{n} c_{i} / \sum_{i=1}^{n} v_{i}$ ) than the system optimum do not want to cooperate. Other agencies could give these higher cost-benefit agencies money to offset the difference between the Nash equilibrium and the social optimum. Alternatively, the government could give these higher cost-benefit ratio agencies some subsidy to entice them to cooperate.

To sum up, we have the investment strategies of agency $1 \& 2$ and the outcomes as shown in Table 5.

In Nash equilibrium, the security level is determined by the agency with the highest cost-benefit ratio, with all the agencies contribute the same, whereas at the social optimum the security level is determined by the cost-benefit ratio of the system $\sum_{i=1}^{n} c_{i} / \sum_{i=1}^{n} v_{i}$, with all agencies contributing the same. The social optimum results in a higher level of security $\left(P^{S}>P^{N}\right)$ and of system utility $\left(U^{S}>U^{N}\right)$.

Imperfect information exclusive node dominated network and mixed network-Combination of total effort and weakest link

If the attacker does not know the current security level of the nodes of an exclusive node dominated network, they will attack randomly, this becomes a partial weakest link case. i.e. $\frac{\partial P}{\partial x_{i}}>\frac{\partial P}{\partial x_{j}}>0$ when $x_{i} \ll x_{j}$. The security function takes the form:

$P\left(x_{1}, x_{2}, \cdots, x_{n}\right) \triangleq \frac{\sum_{i=1}^{n} P\left(x_{i}\right)}{n}=P\left(\sum_{i=1}^{n} a_{i} x_{i}\right)$, where $\sum_{i=1}^{n} a_{i}=1$ and $a_{i}>a_{j}$ if $x_{i}<x_{j}$

The equality holds because of the concavity of $P(\cdot)$

If $n=2$, and $x_{1}<x_{2}$, then $a_{1}>a_{2}$

$P\left(a_{1} x_{1}+a_{2} x_{2}\right)=P\left(\left(a_{1}-a_{2}\right) x_{1}+a_{2} x_{1}+a_{2} x_{2}\right)=P\left(\left(a_{1}-a_{2}\right) \min \left(x_{1}, x_{2}\right)+a_{2}\left(x_{1}+x_{2}\right)\right)$

This is a combination of weakest link and total effort as shown in Figure 5.

Another case that ends in the combination of total effort and weakest link is the mixed network. The network type and the isoquant of security function $P$ are shown in Figure $6 \mathrm{a}$ and $\mathrm{b}$.

Table 5 Nash equilibrium and social optimum of exclusive node dominated network $c_{1} / v_{1}>c_{2} / v_{2}$.

\begin{tabular}{lll}
\hline Nash equilibrium & Social optimum \\
\hline$x_{1}^{N}=x_{2}^{N}=G\left(\frac{c_{1}}{v_{1}}\right)$ & $<x_{1}^{S}=x_{2}^{S}=G\left(\frac{c_{1}+c_{2}}{v_{1}+v_{2}}\right)$ \\
$P^{N}=P\left(G\left(\frac{c_{1}}{v_{1}}\right)\right)$ & $<P^{S}=P\left(G\left(\frac{c_{1}+c_{2}}{v_{1}+v_{2}}\right)\right)$ \\
$U_{1}^{N}=v_{1} P\left(G\left(\frac{c_{1}}{v_{1}}\right)\right)-c_{1} G\left(\frac{c_{1}}{v_{1}}\right)$ & $>U_{1}^{S}=v_{1} P\left(G\left(\frac{c_{1}+c_{2}}{v_{1}+v_{2}}\right)\right)-c_{1} G\left(\frac{c_{1}+c_{2}}{v_{1}+v_{2}}\right)$ \\
$U_{2}^{N}=v_{2} P\left(G\left(\frac{c_{1}}{v_{1}}\right)\right)-c_{2} G\left(\frac{c_{1}}{v_{1}}\right)$ & $<U_{2}^{S}=v_{2} P\left(G\left(\frac{c_{1}+c_{2}}{v_{1}+v_{2}}\right)\right)-c_{2} G\left(\frac{c_{1}+c_{2}}{v_{1}+v_{2}}\right)$ \\
$U^{N}=\left(v_{1}+v_{2}\right) P\left(G\left(\frac{c_{1}}{v_{1}}\right)\right)-\left(c_{1}+c_{2}\right) G\left(\frac{c_{1}}{v_{1}}\right)$ & $<U^{S}=\left(v_{1}+v_{2}\right) P\left(G\left(\frac{c_{1}+c_{2}}{v_{1}+v_{2}}\right)\right)-\left(c_{1}+c_{2}\right) G\left(\frac{c_{1}+c_{2}}{v_{1}+v_{2}}\right)$ \\
\hline
\end{tabular}




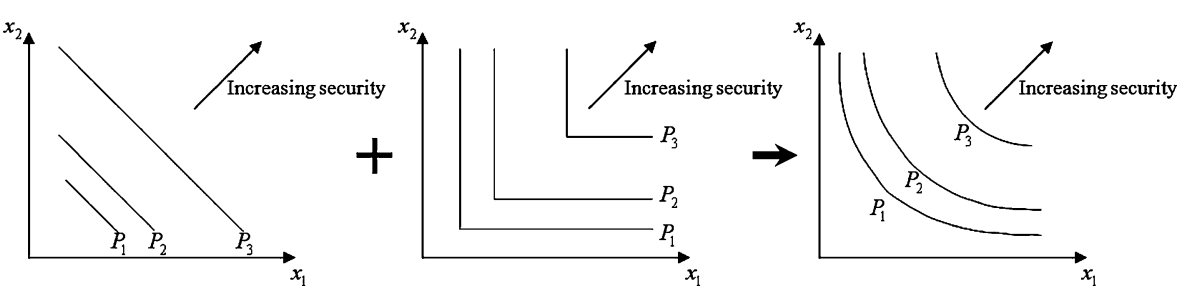

Figure 5 Combination of total effort and weakest link

Since we have social optimum is better than Nash equilibrium in both total effort and weakest link cases, this should also hold for their combination.

\section{Examples}

For a mixed network, one could see that to plan coordinately is better than plan individually. Here we set up a simple topology, two-agency example to illustrate this.

We have two transportation agencies: agency 1 owns node 0 , node 1 ; agency 2 owns node 0 , node 2 . They have a shared node 0 , which is the target, and each has an exclusively owned node, as shown in Figure 7.

Agency 1\&2 want to secure the system at minimum cost. Let $x_{11}, x_{10}$ be the investment on node 1 , node 0 (target) by agency 1 , and $x_{22}, x_{20}$ the investment on node 2 , node 0 by agency 2 . Then the security budget of agency 1 is $x_{1}=x_{10}+x_{11}$, and the security budget of agency 2 is $x_{2}=x_{20}+x_{22}$. The security budget of the system is $x_{1}+x_{2}$.

The attacker could either insert the bomb directly at node 0 (target) or insert the bomb at either node 1 or 2 and make the bomb propagate to node 0 .

Let $p_{0}$ the probability bomb inserted directly at node 0 , and $p_{10}, p_{20}$ the probability bomb inserted indirectly at node 0 from node 1 and node 2 . $p(x)$ should be decreasing since more invest leads to lower bomb insertion rate, and be convex since the more you invest the less you will decrease the probability of bomb insertion. Thus we have

$$
p_{0}=\frac{1}{1+x_{10}+x_{20}}, p_{10}=\frac{1}{1+x_{11}} \frac{1}{1+x_{10}+x_{20}}, p_{20}=\frac{1}{1+x_{22}} \frac{1}{1+x_{10}+x_{20}}
$$

a

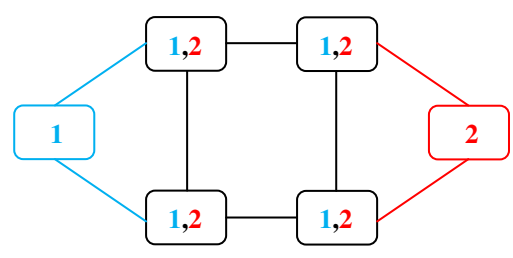

b

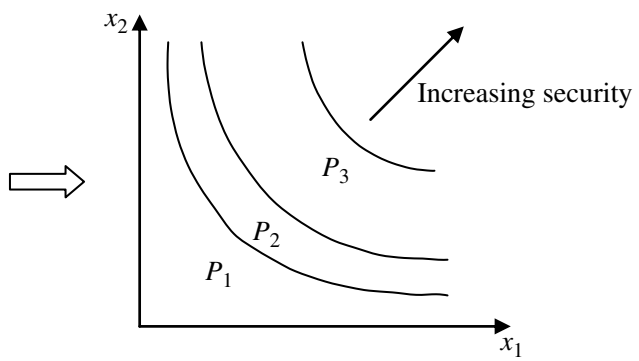

Figure 6 a Mixed network b Isoquant map of security function 


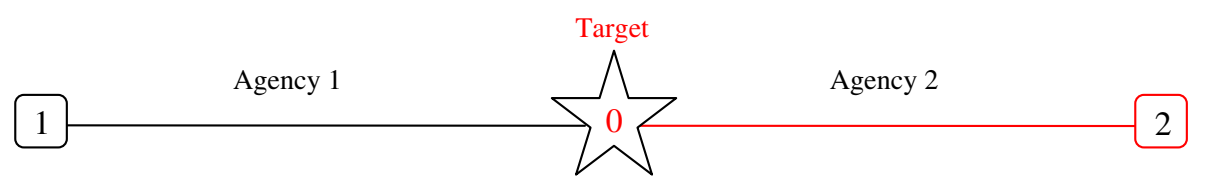

Figure 7 Layout of two agencies

Let $P$ be the probability that the system is secured:

$$
\begin{aligned}
P & =1-\left(p_{0}+p_{10}+p_{20}\right) \\
& =1-\left(\frac{1}{1+x_{1}} \frac{1}{1+x_{10}+x_{20}}+\frac{1}{1+x_{10}+x_{20}}+\frac{1}{1+x_{2}} \frac{1}{1+x_{10}+x_{20}}\right) \\
& =1-\frac{1}{1+x_{10}+x_{20}}\left(1+\frac{1}{1+x_{11}}+\frac{1}{1+x_{22}}\right)
\end{aligned}
$$

Agency $1 \& 2$ each gets $v_{1}, v_{2}$ if the system is secured, their utilities are:

$$
\begin{aligned}
& U_{1}=v_{1} P-x_{1}=v_{1} P-x_{11}-x_{10} \\
& U_{2}=v_{2} P-x_{2}=v_{2} P-x_{22}-x_{20}
\end{aligned}
$$

And the utility of the system $U=U_{1}+U_{2}$.

Similar incentive

If the two agencies have similar incentive to secure the system, let us say $v_{1}=v_{2}=$ 1000. If the two agencies act coordinately under the regulation of the government, they solve $\max _{x_{10}, x_{11}, x_{20}, x_{22}} U$, such that $x_{10}+x_{11}=x_{1}, x_{20}+x_{22}=x_{2}$. From the symmetry of the problem, we know the optimum is reached when $x_{1}=x_{2}, x_{10}=x_{20}$, $x_{11}=x_{22}$. Table 6 shows the optimal investment of each agency on each node when the security budget of the system $\left(x_{1}+x_{2}\right)$ ranges from 5 to 30 .

However, if the two agencies make their investment decision individually, they might lose the holistic view and make decisions superficially because of the complexity of transportation system security. For example, agency 2 might find itself end in higher utility than agency 1 if it chooses to free ride as shown in Table 7

Table 6 Investments, utilities and security level when agency $1 \& 2$ act coordinately.

\begin{tabular}{llllllllll}
\hline $\begin{array}{l}\text { Security } \\
\text { budget }\end{array}$ & $x_{1}$ & $x_{2}$ & $x_{11}$ & \multicolumn{1}{l}{$x_{10}$} & $x_{22}$ & $x_{20}$ & $U_{1}$ & $U_{2}$ & $P$ \\
\hline 5 & 2.5 & 2.5 & 0.464101 & 2.035899 & 0.464103 & 2.035897 & 530.994 & 530.994 & 0.533494 \\
10 & 5 & 5 & 1.123105 & 3.876895 & 1.123106 & 3.876894 & 773.151 & 773.151 & 0.778151 \\
15 & 7.5 & 7.5 & 1.690416 & 5.809584 & 1.690416 & 5.809584 & 854.347 & 854.347 & 0.861847 \\
20 & 10 & 10 & 2.196148 & 7.803852 & 2.196155 & 7.803845 & 892.108 & 892.108 & 0.902108 \\
25 & 12.5 & 12.5 & 2.656867 & 9.843133 & 2.656856 & 9.843144 & 912.7201 & 912.7201 & 0.92522 \\
30 & 15 & 15 & 3.082774 & 11.91723 & 3.082756 & 11.91724 & 925.0082 & 925.0082 & 0.940008 \\
\hline
\end{tabular}


Table 7 Investments, utilities and security level when agency 1 invests, agency 2 free rides.

\begin{tabular}{llllllllll}
\hline Security budget & $x_{1}$ & $x_{2}$ & $x_{11}$ & $x_{10}$ & $x_{22}$ & $x_{20}$ & $U_{1}$ & $U_{2}$ & $P$ \\
\hline 5 & 5 & 0 & 0.436492 & 4.563508 & 0 & 0 & 510.388 & 515.388 & 0.515388 \\
10 & 10 & 0 & 1.000002 & 8.999998 & 0 & 0 & 740 & 750 & 0.75 \\
15 & 15 & 0 & 1.45804 & 13.54196 & 0 & 0 & 819.491 & 834.491 & 0.834491 \\
20 & 20 & 0 & 1.854102 & 18.1459 & 0 & 0 & 857.239 & 877.239 & 0.877239 \\
25 & 25 & 0 & 2.208099 & 22.7919 & 0 & 0 & 877.836 & 902.8362 & 0.902836 \\
30 & 30 & 0 & 2.531131 & 27.46887 & 0 & 0 & 889.8 & 919.8003 & 0.9198 \\
\hline
\end{tabular}

(Note that Table 7 only shows one unwise decision of agency 1 and 2 when they plan individually, it is not a case of Nash equilibrium).

Table 7 shows that the agency that free rides has higher utility than the agency that invests $\left(U_{1}<U_{2}\right)$. This dilemma is more obviously shown in Figure 8 . As a result, both the agencies want to free ride on the system and it ends in responsibility ambiguity (as one Chinese idiom says: one monk fetches the water to drink, two monks have no water to drink).

Compare Tables 6 and 7 for the same security budget of the system $\left(x_{1}+x_{2}\right)$, we could see that when two agencies act coordinately, both of them have higher utility and enjoy higher security level than they act individually. The government plays a significant role in making agencies act coordinately under a holistic view and pulling them out of the dilemma of responsibility ambiguity. This is shown in Figures 8 and 9.

\section{Different incentive}

It is not uncommon in transportation systems that agencies have different incentive to invest in security, some may be indifferent of the damage caused. For example, each airport has shuttle buses serving the passengers, and the bus terminals are usually set close to the airport. While the airport is for all practical purposes

Figure 8 Utilities when two agencies act individually and coordinately

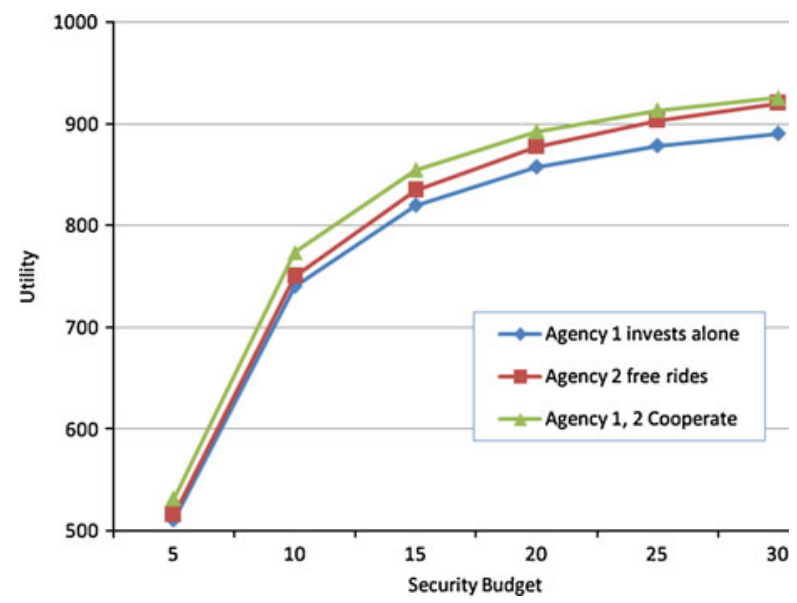


Figure 9 Security levels when two agencies act individually and coordinately

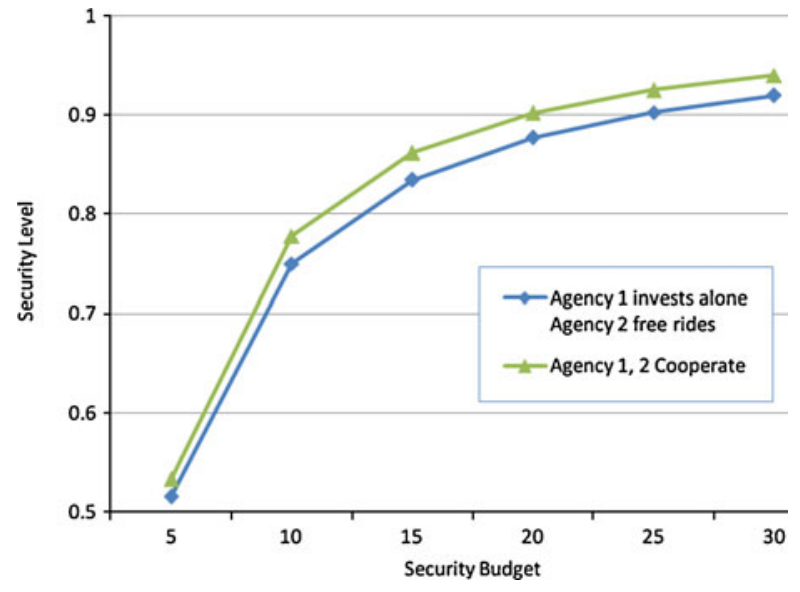

barricaded with layers of security, little or nothing, is done to secure the shuttle buses that oftentimes penetrate deep into its terminal buildings.

Secure the airport alone is not enough while the shuttle bus operator has little incentive to secure its shuttle buses. From the previous example with agency 1 representing airport, $v_{1}=1000$, and agency 2 representing shuttle bus operator, $v_{2}=0$. The shuttle bus operator will always set $x_{20}=x_{22}=0$ since it has no incentive to secure the system. The airport makes investment decision by solving $\max _{x_{10}, x_{11}} U_{1}$, such that $x_{10}+x_{11}=x_{1}, x_{20}=x_{22}=0$. Table 8 shows the Nash Equilibrium when the airport and the shuttle bus operator acts individually under different security budget of the system.

However, this is not the best strategy for the airport, the attacker could easily insert a bomb on a shuttle bus to an airport and the explosion may blow up the whole terminal. It would be better for the airport (agency 1) to pay the shuttle bus operator (agency 2) to secure its buses. In this case, the airport makes investment decision by solving $\max _{x_{10}, x_{11}, x_{20}, x_{22}} U_{1}$, such that $x_{10}+x_{11}=x_{1}, x_{20}+x_{22}=x_{2}$. This is shown in Table 9:

Compare Table 8 with 9 for the same security budget of the system $\left(x_{1}+x_{2}\right)$, we could see that this $1+1$ strategy is better than $2+0$ strategy. The airport ends up in higher utility and enjoys higher security level when it wisely sponsors the shuttle bus operator on its security as shown in Figures 10 and 11.

Table 8 Investments, utilities and security level when agency 2 is indifferent of the damage.

\begin{tabular}{llllllllll}
\hline Security budget & $x_{1}$ & $x_{2}$ & $x_{11}$ & $x_{10}$ & $x_{22}$ & $x_{20}$ & $U_{1}$ & $U_{2}$ & $P$ \\
\hline 5 & 5 & 0 & 0.436492 & 4.563508 & 0 & 0 & 510.388 & 0 & 0.515388 \\
10 & 10 & 0 & 1.000002 & 8.999998 & 0 & 0 & 740 & 0 & 0.75 \\
15 & 15 & 0 & 1.45804 & 13.54196 & 0 & 0 & 819.491 & 0 & 0.834491 \\
20 & 20 & 0 & 1.854102 & 18.1459 & 0 & 0 & 857.239 & 0 & 0.877239 \\
25 & 25 & 0 & 2.208099 & 22.7919 & 0 & 0 & 877.836 & 0 & 0.902836 \\
30 & 30 & 0 & 2.531131 & 27.46887 & 0 & 0 & 889.8 & 0 & 0.9198 \\
\hline
\end{tabular}


Table 9 Investments, utilities and security level when agency sponsors agency 2 to invest on security.

\begin{tabular}{llllllllll}
\hline Security budget & $x_{1}$ & $x_{2}$ & $x_{11}$ & $x_{10}$ & $x_{22}$ & $x_{20}$ & $U_{1}$ & $U_{2}$ & $P$ \\
\hline 5 & 2.5 & 2.5 & 0.463991 & 2.035987 & 0.464036 & 2.035987 & 528.494 & 0 & 0.533494 \\
10 & 5 & 5 & 1.122989 & 3.876979 & 1.123052 & 3.876979 & 768.151 & 0 & 0.778151 \\
15 & 7.5 & 7.5 & 1.690358 & 5.809626 & 1.690391 & 5.809626 & 846.847 & 0 & 0.861847 \\
20 & 10 & 10 & 2.196149 & 7.80385 & 2.196151 & 7.80385 & 882.108 & 0 & 0.902108 \\
25 & 12.5 & 12.5 & 2.656855 & 9.843146 & 2.656853 & 9.843146 & 900.2201 & 0 & 0.92522 \\
30 & 15 & 15 & 3.082766 & 11.91722 & 3.082798 & 11.91722 & 910.0082 & 0 & 0.940008 \\
\hline
\end{tabular}

Figure 10 Utilities when two agencies act individually and coordinately

Figure 11 Security levels when two agencies act individually and coordinately
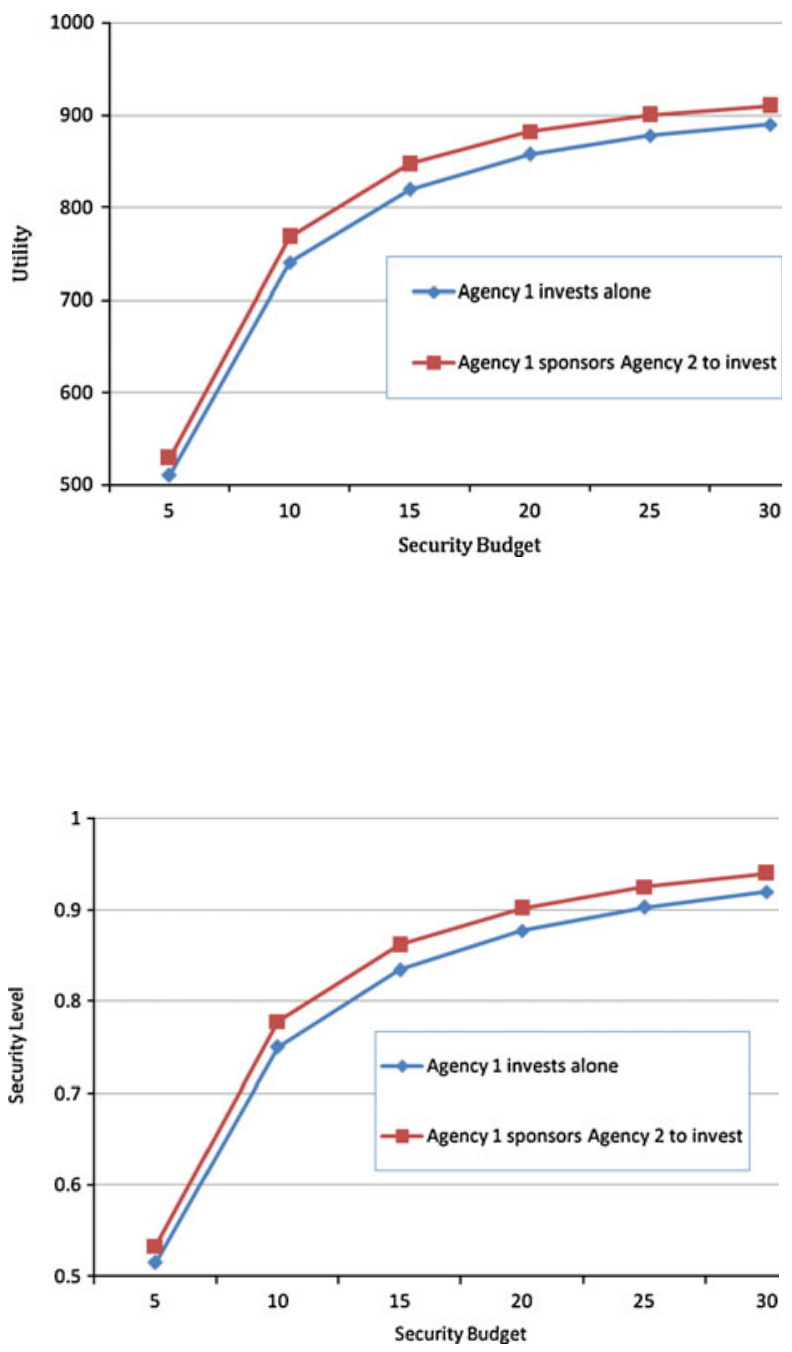


\section{Conclusions}

1. In a multi-agency environment, each agency has its own motivation and effectiveness in the investment on security of the transportation system under its jurisdiction. An agency is motivated to invest on security if its marginal return is larger than marginal cost. Diminishing marginal return and constant marginal cost leads to diminishing utility, thus diminishing incentive to invest. An increase in the investment of one agency is always good for the other agencies dealing with liked transportation systems.

2. In a shared node dominated network, the security level is determined by the advanced agency (the one with either the lowest cost or cost-benefit ratio), other agencies always free ride. Government will allocate the whole security budget to this most advanced agency, thereby letting the most capable and effective agency do everything if the efforts are totally substitutable.

3. In a perfect information exclusive node dominated network, since one agency alone cannot secure the system, each agency only chooses to match the effort of the least capable agency (with highest cost-benefit ratio). In a Nash equilibrium, every agency is dragged down to the performance of the least capable agency. In social optimum, every agency matches the performance of the whole system.

4. In an imperfect information exclusive node dominated network or a mixed network, a combination of total effort and weakest link applies.

5. In all cases, the social optimum is always superior to the Nash equilibrium, resulting in higher security level and sum of utility. The government should mandate the agencies to coordinate or set some incentives to encourage agencies that are not cooperative to coordinate. Alternatively, the more motivated agencies will find it advantageous to pay the less motivated agencies in order to entice them to coordinate.

Open Access This article is distributed under the terms of the Creative Commons Attribution Noncommercial License which permits any noncommercial use, distribution, and reproduction in any medium, provided the original author(s) and source are credited.

\section{References}

Berdica K (2002) An introduction to road vulnerability: what has been done, is done and should be done. Transp Policy 9:117-127

Du ZP, Nicholson A (1997) Degradable transport systems: sensitivity and reliability analysis. Transp Res B 31(3):225-237

Hirshleifer J (1983) From weakest-link to best-shot: the voluntary provision of public goods. Public Choice 41(3):371-386

Jenelius E, Petersen T, Mattsson L-G (2006) Road network vulnerability: identifying important links and exposed regions. Transport Research Arena, Göteborg

Nagurney A, Qiang Q (2007) A transportation network efficiency measure that captures flows, behavior, and costs with applications to network component importance identification and vulnerability. Proceedings of the 18th Annual POMS Conference. Dallas, Texas

Varian HR (2004) System reliability and free riding. Economics of information security. Kluwer, pp 1-15 